phys. stat. sol. 35, 933 (1969)

Subject classification: 18.4

Twente University of Technology, Enschede

\title{
Ground-State Properties of Finite Antiferromagnetic Chains
}

\section{By}

\author{
H. P. van de Braak and W. J. Caspers
}

\begin{abstract}
Exact calculations are presented for the ground state of linear antiferromagnetic Heisenberg chains with open ends. The wave function and the energy are given for chains with $N=4,6$, and 8 , and quantum number $S=1 / 2$. It is shown that in the limit for $N \rightarrow \infty$ the ground state is probably nondegenerate and the long-range order tends to zero. The short-range order shows a strongly oscillating character due to end effects persisting over relatively long distances. A comparison with closed rings is made and the connection with the observation of a reduction of the zero-point deviation in the neighbourhood of non-magnetic impurities in more-dimensional systems is pointed out.
\end{abstract}

Es werden exakte Berechnungen für den Grundzustand einer linearen antiferromagnetischen Heisenbergkette mit offenen Enden mitgeteilt. Die Wellenfunktion und die Energie werden für Ketten mit $N=4,6$ und 8 und der Quantenzahl $S=1 / 2$ angegeben. Es wird gezeigt, daB im Grenzfall $N \rightarrow \infty$ der Grundzustand wahrscheinlich nicht entartet ist und die Fernordnung gegen null geht. Die Nahordnung zeigt einen stark oszillatorischen Charakter, der durch den Einfluß der Enden verursacht wird und über eine relativ große Distanz wirkt. Ein Vergleich mit einem geschlossenen Ring wird durchgeführt und die Verbindung mit der Beobachtung einer Verringerung der Nullpunktsunordnung in der Nähe nichtmagnetischer Verunreinigungen in mehrdimensionalen Systemen aufgezeigt.

\section{Introduction}

The linear antiferromagnetic Heisenberg chain is of great theoretical interest, since it is one of the few quantummechanical manybody systems for which exact calculations are possible. In the isotropic case this system is described by the well-known Hamiltonian

$$
\mathscr{H}=2 \mathscr{J} \sum_{i=1}^{N} \mathbf{S}_{i} \cdot \mathbf{S}_{j},
$$

where $g$ is positive, and usually $S=1 / 2$.

Bethe [1] was the first to derive the ground-state eigenfunction for an infinite chain with periodic boundary conditions. Starting from this solution Hulthén [2] was able to find the ground-state energy. Furthermore he gave explicit values for finite systems consisting of $2,4,6,8$, and 10 spins, and argumented that the total spin quantum number $S$ should be zero. Later on, Lieb and Mattis [3] proved rigorously that the ground state of any finite ring or chain is nondegenerate, which implies of course $S=0$.

The model was generalized to various degrees of anisotropy by Orbach [4] and Walker [5] and applied to finite systems by Bonner and Fischer [6] who did machine calculations for rings with $N=2$ to 11 . In the following we shall refer to the latter paper as B.F. 
In recent years a number of authors have worked out different properties for the model and for a survey we refer to Lieb and Mattis' book [7], which contains also some relevant reprints.

It is not surprising that most attention was paid to the case where periodic boundary conditions are applied since the translational invariance simplifies the calculations considerably. However, the open ended chain has some interesting properties that are worth considering. B.F. indicated that the convergence of the ground-state energy to the asymptotic value for $N \rightarrow \infty$ may be slower than it is in the case of a closed ring, and it is one of the purposes of the present paper to show this to be true indeed.

From previous work of one of the authors [8,9] it appears that the zero-point spin-deviation in two- and three-dimensional Heisenberg antiferromagnets will be reduced in the neighbourhood of a nonmagnetic impurity. Otherwise stated, a pair of neighbouring spins next to such an impurity show a better antiparallel alignment than they would do in a perfect lattice.

In reaching this conclusion some approximations were involved, the most essential of which are the description with spin waves and the assumed short range of the effect. So it will be interesting to know, if this effect will hold in a one-dimensional system, where exact calculations are possible.

It is obvious that the influence of a non-magnetic impurity on a linear ring is adequately accounted for if one studies an open ended chain. However, since the ground state of a finite chain is a singlet, all local magnetizations are zero, and the only way to obtain a rate of short-range order is the calculation of the correlation functions $\left\langle S_{i}^{z} S_{i+1}^{z}\right\rangle$.

For an infinite chain in the Ising limit the ground state is twofold degenerate. This accounts for the fact that it is impossible to build up a finite sublattice magnetization.

This degeneracy, however, is split by the other terms in the Hamiltionian, but the splitting is known to arise only in $N$-th order perturbation theory. So it might be expected that in the limit $N \rightarrow \infty$ the ground state will again become degenerate [6].

The third purpose of this paper is therefore the calculation of the first excited state for finite chains and rings and to analyse if the gap between the ground state and the first excited state suggests a degeneracy in the limit $N \rightarrow \infty$.

Practical considerations restricted the size of the chain to 8 spins, since lack of translational invariance involves, that the number of reductions of the secular determinant is small. In fact the only relevant reduction arises from the singlet character of the ground state, which in the case of $N=8$ leaves a $10 \times 10$ matrix to be diagonalized. However, no really new characteristics are to be expected for larger finite systems, as can be concluded from B.F.'s calculations for periodic rings [6].

\section{Derivation and Solution of the Secular Equation}

We consider a linear chain of spins $1 / 2$ coupled by the Hamiltonian

$$
\mathscr{H}=2 \sum_{i=1}^{N-1} \mathbf{S}_{i} \cdot \mathbf{S}_{i+1} \cdot
$$

For convencience we put the exchange constant $y=1$. It should be observed that $S^{z}=\sum_{i} S_{i}^{z}$ commutes with the Hamiltionan and so does $\boldsymbol{S}^{2}=\left(\sum_{i} \boldsymbol{S}_{i}\right)^{2}$ 
for this particular isotropic case. Reversal of the order of the spins leaves the Hamiltonian unchanged, so parity for order reversal must also be a constant of motion. Bearing these facts in mind we can construct the eigenfunctions starting from 2-spin states for suitably chosen pairs using the general rules of addition of angular momenta. The case $N=2$ is trivial. We find a singlet with odd parity, denoted by $(0-)$, and a triplet with even parity, denoted by $(1+, m)$ where $m=-1,0,+1$. The energy eigenvalues are $-3 / 2$ and $1 / 2$ respectively.

For $N=4$ we consider first the two pairs $(1,4)$ and $(2,3)$ which can be in the singlet or the triplet state, and combine these states into 4-spin states that are eigenfunctions of $S^{z}$ and $\boldsymbol{S}^{2}$. By construction they are also eigenstates of the order reversal operator. The result is

$$
\begin{aligned}
(0+; 1) & =(0-)_{14}(0-)_{23}, \\
(0+; 2) & =\sum_{\mu}\langle 11 \mu-\mu \mid 00\rangle(1+, \mu)_{14}(1+,-\mu)_{23}, \\
(1-, m ; 1) & =(0-)_{14}(1+, m)_{23}, \\
(1-, m ; 2) & =(1+, m)_{14}(0-)_{23}, \\
(1+, m) & =\sum_{\mu}\langle 11 \mu m-\mu \mid 1 \quad m\rangle(1+, \mu)_{14}(1+, m-\mu)_{23}, \\
(2+, m) & =\sum_{\mu}\langle 11 \mu m-\mu \mid 2 m\rangle(1+, \mu)_{14}(1+, m-\mu)_{23} .
\end{aligned}
$$

The symbol $\left\langle j_{1} j_{2} m_{1} m_{2} \mid j m\right\rangle$ stands for a Clebsch-Gordon coefficient [10]. All these states will be important in the cases of higher $N$-values, but for the moment we are looking for the ground state, so only the first two are relevant, being singlets. In a more explicit form we have

$$
\begin{gathered}
(0+; 1)=\frac{1}{2}[(++--)-(+-+-)-(-+-+)+(--++)], \\
(0+; 2)=\frac{1}{2 \sqrt{3}}[-(++--)-(+-+-)+2(+--+)+ \\
+2(-++-)-(-+-+)-(--++)] .
\end{gathered}
$$

The calculation of matrix elements of $\mathscr{H}$ is greatly simplified by the time-reversal symmetry of the state vectors and one easily finds the following secular matrix

$$
\begin{array}{c|cc} 
& (0+; 1) & (0+; 2) \\
\hline(0+; 1) & -\frac{3}{2} & -\sqrt{3} \\
(0+; 2) & -\sqrt{3} & -\frac{3}{2}
\end{array}
$$

The eigenvalues are $\varepsilon_{0,1}=-3 / 2 \pm \sqrt{3}$ and the ground-state eigenfunction is

$$
\psi_{0}^{4}=\frac{1}{\sqrt{2}}[(0+; 1)+(0+; 2)] .
$$

A pplication of periodic boundary conditions would require an additional term $2 \boldsymbol{S}_{4} \cdot \boldsymbol{S}_{1}$ in the Hamiltionian. Since both $(0-)_{14}$ and $(1+, m)_{14}$ are eigenfunc- 
tions of this operator, the effect on the secular matrix is confined to the diagonal terms, which turn out to be -3 and -1 in this case. The eigenvalues are now

$$
\varepsilon_{0}=-4 \text { and } \varepsilon_{1}=0
$$

and the ground state is

$$
\varphi_{0}^{4}=\frac{\sqrt{3}}{2}(0+; 1)+\frac{1}{2}(0+; 2) .
$$

For the case $N=6$ we start from the 4 -spin states for the system $(16 ; 25)$, which are already known, and combine these with $(0-)_{34}$ or $(1+)_{34}$ so as to obtain singlets. The result is

$$
\begin{aligned}
(0-; \alpha)= & (0-)_{16}(0-)_{25}(0-)_{34}, \\
(0-; \beta)= & \sum_{\mu}\langle 11 \mu-\mu \mid 00\rangle(1+, \mu)_{16}(1+,-\mu)_{25}(0-)_{34}, \\
(0-; \gamma)= & \sum_{\mu}\langle 11 \mu-\mu \mid 00\rangle(0-)_{16}(1+, \mu)_{25}(1+,-\mu)_{34}, \\
(0-; \delta)= & \sum_{\mu}\langle 11 \mu-\mu \mid 00\rangle(1+, \mu)_{16}(0-)_{25}(1+,-\mu)_{34}, \\
(0+)= & \sum_{m, \mu}\langle 11 m-m \mid 00\rangle\langle 11 \mu m-\mu \mid 1 m\rangle \times \\
& \times(1+, \mu)_{16}(1+, m-\mu)_{25}(1+,-m)_{34} .
\end{aligned}
$$

We note that the ground state will either be $(0+)$ or a linear combination of the $(0-)$ 's. The singlets with odd parity are all build up from a 4-spin singlet and a singlet for the third pair, so the explicit form can be derived easily from the foregoing case $N=4$. We shall not go into the details but merely give the secular matrix:

\begin{tabular}{c|cccc} 
& $(0-; \alpha)$ & $(0-; \beta)$ & $(0-; \gamma)$ & $(0-; \delta)$ \\
\hline$(0-; \alpha)$ & $-\frac{3}{2}$ & $-\sqrt{3}$ & $-\sqrt{3}$ & 0 \\
$(0-; \beta)$ & $-\sqrt{3}$ & $-\frac{7}{2}$ & 0 & 1 \\
$(0-; \gamma)$ & $-\sqrt{3}$ & 0 & $-\frac{3}{2}$ & 1 \\
$(0-; \delta)$ & 0 & 1 & 1 & $\frac{1}{2}$
\end{tabular}

The lowest two eigenvalues are

$$
\varepsilon_{0}=-4.98715 \text { and } \varepsilon_{1}=-2.37045,
$$

whereas the eigenvalue of the state $(0+)$ equals $-4 / 3$, so it is definitely not the ground state.

If we write $\psi_{0}^{6}=\sum_{\nu} a_{v}(0-; v)$, we find

$$
\begin{array}{ll}
a_{\alpha}=0.53537, & a_{\gamma}=0.32226 \\
a_{\beta}=0.75561, & a_{\delta}=-0.19643 .
\end{array}
$$


For the case with periodic boundary conditions the diagonal elements of the secular matrix turn out to be $-3,-3,-3$, and 1 .

The corresponding lowest two eigenvalues are

$$
\varepsilon_{0}=-5.60555 \text { and } \varepsilon_{1}=-3.0000 .
$$

The state vector $\varphi_{0}^{6}$ is determined by the coefficients

$$
\begin{array}{ll}
b_{\alpha}=0.67676, & b_{\gamma}=0.50904, \\
b_{\beta}=0.50904, & b_{\delta}=-0.15413 .
\end{array}
$$

The construction of the angular momentum eigenfunctions that form a suitable basis for the 8-spin problem goes along similar lines. We divide the systems into two subsystems $(18 ; 27)$ and $(36 ; 45)$ for which the functions are already given above, and combine these states into 8-spin singlets. For the sake of shortness we shall not write those down, but indicate how they are build up. First there is the state in which all four pairs are singlets. There are six possibilities for which we have two triplet pairs combining into a 4 -spin singlet, while the remaining two pairs are singlets. The last three states are those in which every pair is a triplet that are combined to 4-spin singlets, triplets and quintets respectively. Those 4 -spin states will finally result in 8 -spin singlets. All these 10 singlets for the 8-spin system are of even parity, as could be expected. (There are also two singlets of odd parity, but they can be disregarded as far as the ground state is concerned.) The explicit form of some of the state vectors can again rather easily be derived from the results for $N=6$, by simply adding one pair in a singlet state. Diagonalization of the secular matrix was performed by a machine calculation. The relevant eigenvalues are

$$
\varepsilon_{0}=-6.74987 \quad \text { and } \quad \varepsilon_{1}=-4.66761 \text {. }
$$

For the periodic case these values are

$$
\varepsilon_{0}=-7.30219 \quad \text { and } \quad \varepsilon_{1}=-5.39926 .
$$

\section{Energy of the Ground State}

In this section we shall analyse the dependence of the energy of the ground state upon $N$ and investigate the behaviour of the splitting between ground state and first excited state. It is convenient to normalize the energy by dividing it by the number of neighbour pairs in the chain.

In Table 1 we summarize the results both for open chains and closed rings, and for completeness the values obtained by Hulthén [2] are shown as well.

Table 1

Energy of the antiferromagnetic ground state

\begin{tabular}{c|c|c|c}
\hline $\mathrm{N}$ & Open chain & $\begin{array}{c}\text { Closed ring } \\
\text { (present work) }\end{array}$ & $\begin{array}{c}\text { Closed Ring } \\
\text { (Hulthén) }\end{array}$ \\
\hline 2 & -1.50000 & -1.50000 & -1.5000 \\
4 & -1.09402 & -1.00000 & -1.0000 \\
6 & -0.99743 & -0.93426 & -0.9343 \\
8 & -0.96427 & -0.91277 & -0.9128 \\
$\vdots$ & & & \\
$\infty$ & & $-0.88629 *)$ & -0.8875
\end{tabular}

*) This value is due to Orbach [4]. 


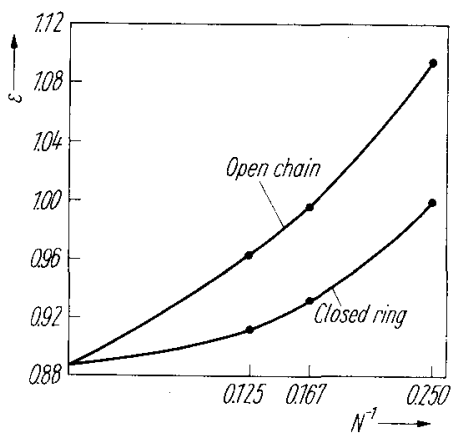

Fig. 1. The energy of the ground state of antiferromagnetic chains and rings as a function of the number of spins

It may be observed that the values for the closed rings show excellent agreement with the results in B.F. [6]. These authors suggested that the convergence of the energy to its limiting value for $N \rightarrow \infty$ would be slower for the open chain. A more careful analysis shows that there is a tendency for the energy to become linear with $N^{-1}$ for large $N$-values. For the closed chain the dependence is likely to be quadratic.

In Section 1 we mentioned the problem of the asymptotic degeneracy of the ground state. In this context it would be illuminating to plot the splitting $\Delta \varepsilon=\varepsilon_{1}-\varepsilon_{0}$ as a function of $N$.

However, even in a non-degenerate case the levels will close up to a continuum for $N \rightarrow \infty$. In the tails of the distribution of energy levels one would expect a density roughly proportional to $N^{-1}$, so a useful criterium for asymptotic degeneracy would be a decrease of $N \cdot \Delta \varepsilon$ with increasing $N$.

\section{Table 2}

Dependence of the splitting of the ground state upon $N$

\begin{tabular}{c|c|c}
\hline \multirow{2}{*}{$N$} & \multicolumn{2}{|c}{$N \cdot \Delta \varepsilon$} \\
\cline { 2 - 3 } & Open chain & Closed ring \\
\hline 4 & 13.85640 & 16.00000 \\
6 & 15.70020 & 15.63330 \\
8 & 16.65708 & 8.74344
\end{tabular}

From Table 2 one sees clearly that the criterium is fulfilled for the closed rings. At this point our results disagree with those by B.F. [6], who conclude that in the perfectly isotropic Heisenberg ring no degeneracy will occur and no stable sublattice magnetization can be built up. It is remarkable, however, that according to the above criterium the open chain has an essentially different character, since $N \cdot \Delta \varepsilon$ increases in this case. A possible maximum at a certain- $N$-value, which would of course remove this descrepancy, is not very likely to occur. In this light it would be interesting to try and find analytical results for the open ehain in the limit for $N \rightarrow \infty$. 


\section{Correlations in the Ground State}

Order parameters for a magnetic system are conveniently defined by

$$
\omega_{i j}=\left\langle S_{i}^{z} S_{j}^{z}\right\rangle .
$$

These quantities are easily accessible for investigation since we know the state vector of the ground state in a representation particularly suitable for the calculation of matrix elements of $S_{i}^{z} S_{j}^{z}$. The most interesting cases for our purpose are $j=i+1$, corresponding to short-range order, and $i=1, j=N$, corresponding to long-range order. For closed rings all short-range order parameters are equal by translational invariance, but, as pointed out in Section 1 , this is not to be expected for an open chain.

The definition of long-range order, as we use it here, does not apply to closed rings, but for open chains it is the most natural choice. We present the results in Table 3.

Table 3

The ground-state correlation function $\omega_{i j}(N)$

\begin{tabular}{|c|c|c|c|c|c|c|}
\hline \multicolumn{6}{|c|}{ Open chain } & Closed ring \\
\hline$N$ & $\omega_{12}$ & $\omega_{23}$ & $\omega_{34}$ & $\omega_{45}$ & $\omega_{1 N}$ & $\omega_{i i+1}$ \\
\hline 2 & -0.25000 & & & & -0.25000 & -0.25000 \\
\hline 4 & -0.22767 & -0.08333 & & & -0.08333 & -0.16667 \\
\hline 6 & -0.22249 & -0.09185 & -0.20252 & & -0.04682 & -0.15571 \\
\hline 8 & -0.22041 & -0.09476 & -0.19579 & -0.10307 & -0.03107 & -0.15213 \\
\hline
\end{tabular}

The values for the closed ring are again in excellent agreement with B.F. and the open chain results for $N=6$ come close to those obtained by Lieb, Schultz, and Mattis in their paper on the $X Y$-model [11].

We note that the long-range order parameter for the open chain tends to zero for large $N$, which is perhaps not surprising in the light of the conclusion that in this limit the ground is probably not degenerate.

Furthermore it is clear that the correlation function has a larger value at the end of a chain $\left(\omega_{12}\right)$ than for the next pair $\left(\omega_{23}\right)$. This effect gives strong support for the idea, that even in a three-dimensional lattice two spins next to a nonmagnetic impurity tend to line up antiparallelly even better than in a perfect lattice. In this one-dimensional case the effect is of course exaggerated because of the smaller number of nearest neighbours causing the normal zero-point deviation. It is remarkable, however, to observe that the oscillating character of the short-range order seems to persist even at large distances from the end. There is also no clear tendency to weaker oscillation for larger $N$-values. This again suggests that exact calculations for an infinite open Heisenberg chain might possibly lead to unexpected properties.

\section{Acknowledgements}

The authors are indebted to Dr. J. R. Luyten of the Computer Centre, University of Groningen, for his help with calculations. 


\section{References}

[1] H. A. Bethe, Z. Phys. 71, 205 (1931).

[2] L. Hulthén, Ark. Mat. Astr. Fys. (Sweden) 26A, No. 11, 1 (1938).

[3] E. Lieb and D. MatTis, J. Math. Phys. 3, 749 (1962).

[4] R. Orbach, Phys. Rev. 112, 309 (1958).

[5] L. R. Walker, Phys. Rev. 116, 1089 (1959).

[6] J. C. Bonner and M. E. Fischer, Phys. Rev. 135, A460 (1964).

[7] E. Lieb and D. Matris, Mathematical Physies in One Dimension, Academic Press, 1966.

[8] H. P. van De BraAk, Thesis, University of Groningen, 1968.

[9] H. P. van de Braak, Physica 42, 137 (1969).

[10] E. U. Condon and G. H. Shortuey, The Theory of Atomic Spectra, Cambridge University Press, 1957.

[11] E. Limb, T. Schultz, and D. Mattis, Ann. Phys. (USA) 16, 407 (1961).

(Received July 7, 1969) 